C-A/AP/\#153

June 2004

\title{
Use of maps in the exploration of electron cloud parameter space
}

U. Iriso and S. Peggs

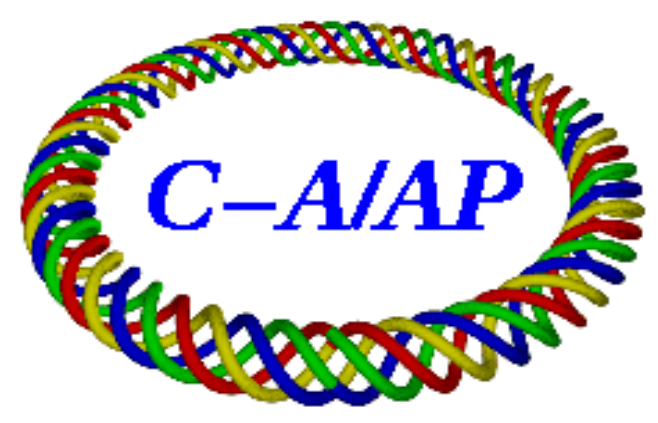

Collider-Accelerator Department Brookhaven National Laboratory Upton, NY 11973 
$\mathrm{CAD} / \mathrm{AP} / 153$

June 10, 2004

\title{
Use of maps in the exploration of electron cloud parameter space
}

\author{
U. Iriso and S. Peggs
}

\begin{abstract}
The optimal distribution of the bunch pattern around the RHIC circumference is studied to decrease the electron density for a fixed total beam current. In the search for a bunch pattern that minimizes this density, we show that, for typical parameters, the bunch-to-bunch evolution of the electron cloud density can be represented by a cubic map. Furthermore, we discuss the use of linear bunch-to-bunch maps for small electron cloud densities. The linear coefficients evaluate the electron cloud stability for a given set of physical parameters (bunch charge, SEY, et cetera). Thus, the use of (linearized) maps frees up the slow but detailed simulation codes to explore parameter space, when studying the increase (to a saturated value) or disappearance of the electron cloud under alternative bunch patterns.
\end{abstract}

\section{Motivation}

Electric fields present in many vacuum systems may accelerate electrons (produced by field emission, photoemission, ionization of residual gas ionization, et cetera) towards the wall chamber surface. If the bombarding electrons acquire enough energy, they produce secondary electrons, which in turn may be accelerated if the electric field normal to the surface is in the correct phase. These electrons may bombard another surface and emit again secondary electrons. This bouncing back and forth between surface is the electron multipacting effect. This name is derived from "resonance of multiple electron impact", and it was first described by Farnswoth in 1934 [1]. If the ratio between the number of outgoing electrons per impinging electron, given by the Secondary Emission Yield (SEY) of the wall surface, is greater than unity, the electron density inside the pipe increases (initially) exponentially, creating a so-called Electron Cloud (EC). This EC can eventually lead to the development of vacuum breakdown and other system failures.

Since 1965, when a coherent betatron oscillation and beam loss in presence of bunched beams were observed in BINP in Novosibirsk [2], footprints of electron multipacting produced by the beam electric field have been detected in many accelerators. In 1972, the ISR at CERN showed excitation of nonlinear resonances and gradual beam blow up in presence of proton coasting beams, with a beam induced signal from a pick up showing coupled electron-proton oscillations [3]. After a couple of decades, electron driven instabilities started to be an issue in several machines (see Reference [4] for an historical overview on Electron Cloud effects), and finally, in 1997 CERN launched a crash program to study the effects of the Electron Cloud due to its important impact on the LHC.

Several computer codes have been successfully developed and benchmarked with experimental observations since the late nineties to study the build up conditions of this effect. A comparison among the different codes was made [5] after the "Eletron Cloud Workshop02" held in Geneva in 2002 [6]. Typically, these codes work either by Particle In Cell methods (like CLOUDLAND [7]), or by tracking the electrons grouped in macro-particles, where each macro-particle can join up to a maximum of around $10^{5}$ electrons (like ECLOUD [8], or CSEC [9]). In the latter case, when a macro-particle produces more electrons, the total Coulomb charge of this macro-particle is increased. At every time step, these detailed codes compute the necessary physical forces/fields influencing the motion of the macro-particles. If EC formation takes place, the total number of electrons is about $10^{10}$ (depending on the parameters for every case), hence the need to group the electrons in macro-particles. These codes use a considerable amount of CPU time: a complete EC simulation, depending specially on the simulation parameters, can last from around 1 hour to some days. In the cases we studied here (for the parameters seen in Table 1), a single simulation last about 1 hour. 


\section{The bunch to bunch evolution}

In case of a multi-bunch electron cloud, where the electric field accelerating the electrons is given by a bunched beam, it is postulated that the evolution of the electron cloud density can be followed using logistic maps. This frees up the detailed simulation codes and enhances physical intuition through the use of standard maths. For a given beam pipe characteristics (SEY, chamber dimensions, etc), the evolution of the electron density, $\rho$, is only driven by the bunch $m$ passing by, and the existing electron density before the bunch passed by. Following the logistic map formalism, this would be expressed as:

$$
\rho_{m+1}=\alpha \rho_{m}\left(1-\rho_{m}\right),
$$

and the parameter $\alpha$ would be a first function of the beam parameters, such as bunch intensity, $N$; bunch spacing, $s_{b}$; bunch length, $\sigma_{z}$; and bunch transverse size, $r_{b}$. Ultimately, $\alpha$ would be a function of the beam pipe characteristics: maximum SEY, $\delta_{\max }$; electron energy at which SEY is maximum, $E_{\text {max }}$; reflectivity at zero electron energy, $R_{0}$; beam pipe dimensions, etc:

$$
\alpha=\alpha\left(N, s_{b}, \sigma_{z}, r_{b}, \ldots ; \delta_{\max }, E_{\max }, R_{0}, \ldots\right) .
$$

Therefore, $\alpha$ would be a mathematical tool concentrating the EC dependence of the physical parameters. Note that the units in Eq. 1 do not fit, and requires $\rho_{m}$ to be unit-less. Equation 1 is only a mathematical tool to express our purposes. This formalism evidences one of the main known characteristics of the electron cloud, the initial exponential growth [13]:

$$
\rho \approx \rho_{0} e^{m(\alpha-1)},
$$

is now explained with Eq. 1. From the latter expression, the saturated electron cloud density, $\rho_{\text {sat }}$ is determined simply by:

$$
\begin{aligned}
& \rho_{\text {sat }}=0 ; \quad \alpha \leq 1, \\
& \rho_{\text {sat }}=\frac{\alpha-1}{\alpha} \quad ; \quad \alpha \geq 1,
\end{aligned}
$$

showing a phase transition from electron cloud "off" to "on". If $\alpha$ increases smoothly with $N$, the phase transition is second order. However, RHIC data shows both first and second order electron cloud phase transitions [14]. Although the logistic map formalism is finally not appropriate, it illustrates the purpose of this study: simplify the EC problem into a small number of mathematical parameters. In the example of the logistic maps, the sole parameter is $\alpha$.

We then tackle the problem by testing if the existing computer simulations confirm that the electron cloud evolution can be represented by maps. For this purpose, we center the following studies on the RHIC case. Table 1 shows the physical parameters used for these simulations. Besides the beam characteristics, the SEY behavior as a function of the impinging electron energy is a key parameter in the electron cloud development. All simulation codes are strongly dependent of the model used for the SEY behavior [10]. In this case, CSEC uses the model by M. Furman described in [11], where one can find detailed explanations of the parameters named in the second part of Table 1. On the other hand, ECLOUD uses the model described in [10]. Table 1 compares only the most "common" surface physics parameters. It is worth mentioning that whereas $E C L O U D$ uses a Gaussian distribution for the emitted secondary electrons, CSEC uses a Lorentzian one (parameter $\sigma_{\text {sec }}$ in Table 1 ).

Note that with this bunch spacing and given the RHIC revolution period, we can inject up to 120 bunches (not counting the limitations given by the abort gap kickers, which would decrease this number to 112). Nevertheless, for the purpose of this study we are interested in the build up and decay evolution of the electron density. Therefore, launching the simulation until the saturation is reached, (which is well achieved after 60 consecutive bunches) minimizes the CPU time.

A typical evolution of the electron density is shown in Figure 1. This corresponds to the case of injecting 60 bunches of charge $1.4 \cdot 10^{11}$ protons in the ring spaced by the minimum RHIC bunch spacing (108 ns). The electron density per beam pipe meter $(\rho)$ as a function of time grows exponentially until the space charge due to the electrons themselves produces a saturation level. In this case, the code used is CSEC (Cylindrical Symmetric Electron Cloud simulation) [12]. The red line shows CSEC output, while the grey circles mark the average electron density between the passage of two bunches, no matter if they are full bunches or empty. As expected, once the saturation level is reached the average electron density does not change significantly. 
Table 1: List of input parameters for electron cloud simulations.

\begin{tabular}{lcccc}
\hline \hline & & & $C S E C$ & $E C L O U D$ \\
\hline parameter & symbol & unit & value & value \\
\hline bunch spacing & $s_{b}$ & $\mathrm{~ns}$ & 108 & 108 \\
\# of bunches & $M$ & $\ldots$ & 60 & 60 \\
beam radius & $r_{b}$ & $\mathrm{~mm}$ & 2.4 & 2.4 \\
full bunch length & $\sigma_{z}$ & $\mathrm{~ns}$ & 18 & 21 \\
bunch charge & $N$ & $\mathrm{p} \cdot 10^{10}$ & 8 to 20 & 8 to 20 \\
revolution time & $t_{r e v}$ & $\mu s$ & 12.82 & 12.79 \\
relativistic factor & $\gamma$ & $\ldots$ & 26 & 10.75 \\
\hline beam pipe diameter & $d$ & $\mathrm{~mm}$ & 60 & 60 \\
reflectivity at zero energy & $R_{0}$ & $\ldots$ & 0.6 & 1.0 \\
reflectivity at infinite energy & $P_{\infty}$ & $\ldots$ & 0.2 & $\ldots$ \\
rediffusion probability & $P_{r d}$ & $\ldots$ & 0.5 & $\ldots$ \\
reflection energy & $E_{r f}$ & $\mathrm{eV}$ & 60 & 60 \\
maximum SEY & $\delta_{\max }$ & $\ldots$ & 2.3 & 2.3 \\
energy for maximum SEY & $E_{\max }$ & $\mathrm{eV}$ & 310 & 310 \\
energy for secondary e- & $E_{\text {sec }}$ & $\mathrm{eV}$ & 8.9 & 7.0 \\
energy width for secondary e- & $\sigma_{\text {sec }}$ & $\mathrm{eV}$ & 4.5 & 5.5 \\
\hline initial e- density & $\rho_{c e}$ & $\mathrm{pC} \cdot \mathrm{m}$ & 0.2 & - \\
electrons generated/bunch & $\ldots$ & $\ldots$ & 35000 & - \\
electron generation radius & $\ldots$ & $\mathrm{mm}$ & 60 & - \\
\hline \# slices per bunch & $\ldots$ & $\ldots$ & 60 & 100 \\
\# slices per inter-bunch & $\ldots$ & $\ldots$ & 840 & 100 \\
initial \# of macro-particles & $\ldots$ & $\ldots$ & 25 & - \\
maximum \# of macro-particles & $\ldots$ & $\ldots$ & $10^{5}$ & $\approx 10^{5}$ \\
\hline \hline
\end{tabular}

Figure 1 (bottom) shows two zooms for the build up and decay time in Fig. 1. Obviously, in the bunch to bunch evolution, the time step is now integer multiples of the bunch spacing. Taking this fact into account, Fig. 1 shows that following the evolution "bunch-to-bunch" does not produce a lack of information about the build-up or the decay time, although the details of the electron density oscillation between two bunches are lost.

\section{The cubic map}

Using the parameters shown in Table 1, the bunch to bunch evolution of the electron cloud density is followed averaging the output of two codes, CSEC and ECLOUD, for different bunch intensities, $N$, from $6 \cdot 10^{10} \mathrm{p}$ to $2 \cdot 10^{11} \mathrm{p}$, in steps of $\Delta N=2 \cdot 10^{10} \mathrm{p}$. Figure 2 shows how the electron density after the bunch $m$ passes by, $\rho_{m+1}$, behaves as a function of the previous electron density, $\rho_{m}$, for different bunch intensities, $N$. The points in Fig. 2 show the average electron cloud density between two bunches using results from CSEC (Fig. 2, left) and ECLOUD (Fig. 2, right). The lines correspond to cubic fits with no constant term:

$$
\rho_{m+1}=a \rho_{m}+b \rho_{m}^{2}+c \rho_{m}^{3} .
$$

Figure 2 is explained as follows: starting with a small initial linear electron density $\rho_{0} \neq 0$ (due to beamgas ionization, beam losses, etc), after some bunches the density takes off and reaches the corresponding saturation line $\left(\rho_{m+1}=\rho_{m}\right.$, red trace) when the space charge effects due to the electrons of the cloud itself takes place. In this situation, all the points (corresponding to the passage of full bunches) are in the same spot. The justification of the three terms is explained as a consequence of the linear growth, a parabolic decay due to space charge effects, and a cubic term corresponding to perturbations (electrons generated by residual gas ionization, beam losses, etc).

The electron cloud decay is described as the succession of bunches with a null bunch intensity, $N=0$. Neglecting the point corresponding to the electron cloud density after the first empty bunch, the electron density follows a similar decay independently of the initial value of the saturated electron density.

It is worth stressing the behavior of this "first empty" bunch, corresponding to the $N=0$ bunches. The 

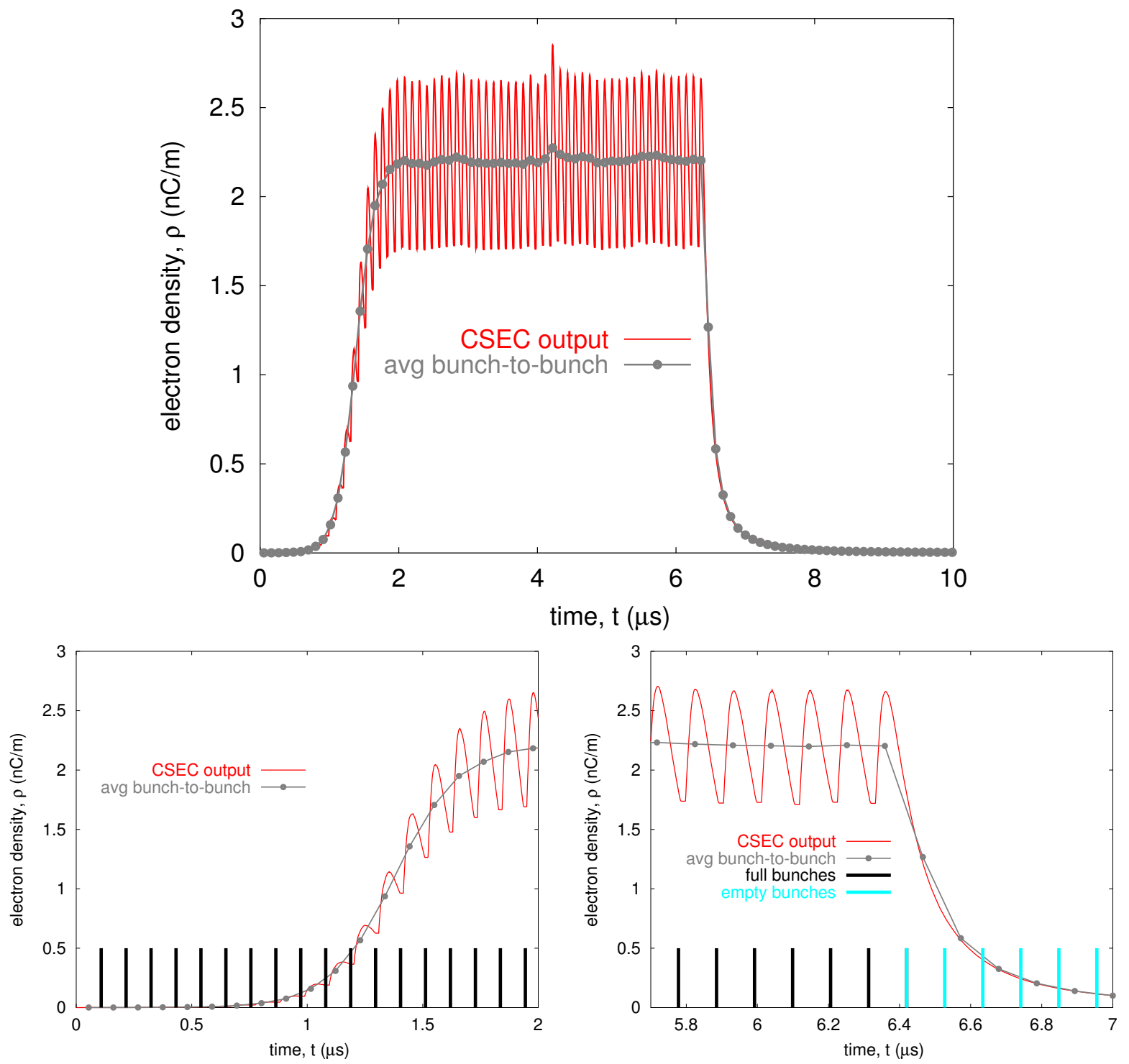

Figure 1: Top: CSEC output of an electron cloud build-up in RHIC as a function of time (red line) during $9 \mu s$ a (RHIC revolution is $(12.82 \mu s)$. The grey circles mark the average electron density between two bunches. Bottom: The left plot shows a zoom of the electron cloud build up shown in the top plot, which takes place after the passage of 18 consecutive bunches (black bars). The plot on the right shows a zoom for the electron cloud decay during the passage of 12 bunches: 6 of them are full bunches (with bunch charge $N=1.4 \cdot 10^{11} \mathrm{p}$, black bars), and six of them correspond to "empty" bunches (bunches with bunch charge $N=0 \mathrm{p}$, light blue bars).

points coming from different saturation values, $\rho_{\text {sat }}$ lye off on a general curve, which we call "first $N=0$ ", or "first empty bunch" curve. This is explained as a consequence of the dominance of the space charge effects of the saturation. In other words, it takes two bunches to jump from a curve $N \neq 0$ to the decay $(N=0$ curve).

Thus, for the parameters shown in Table 1, the electron density build up for a given bunch intensity is determined by a 3-dimensional vector $\vec{A}(N)=(a, b, c)$, while decay is described by two vectors, one corresponding to the "first ghost bunch", and a second vector for the rest of them. Figure 3 shows how the coefficients $(a, b, c)$ evolve as a function of the bunch intensity, $N$, for both $C S E C$ (grey points), and $E C L O U D$ (red squares). Explanation of the physical meaning of these parameters follows. First, for this bunch spacing and surface parameters, both codes give a similar phase transition threshold, around $7 \cdot 10^{10}$ 

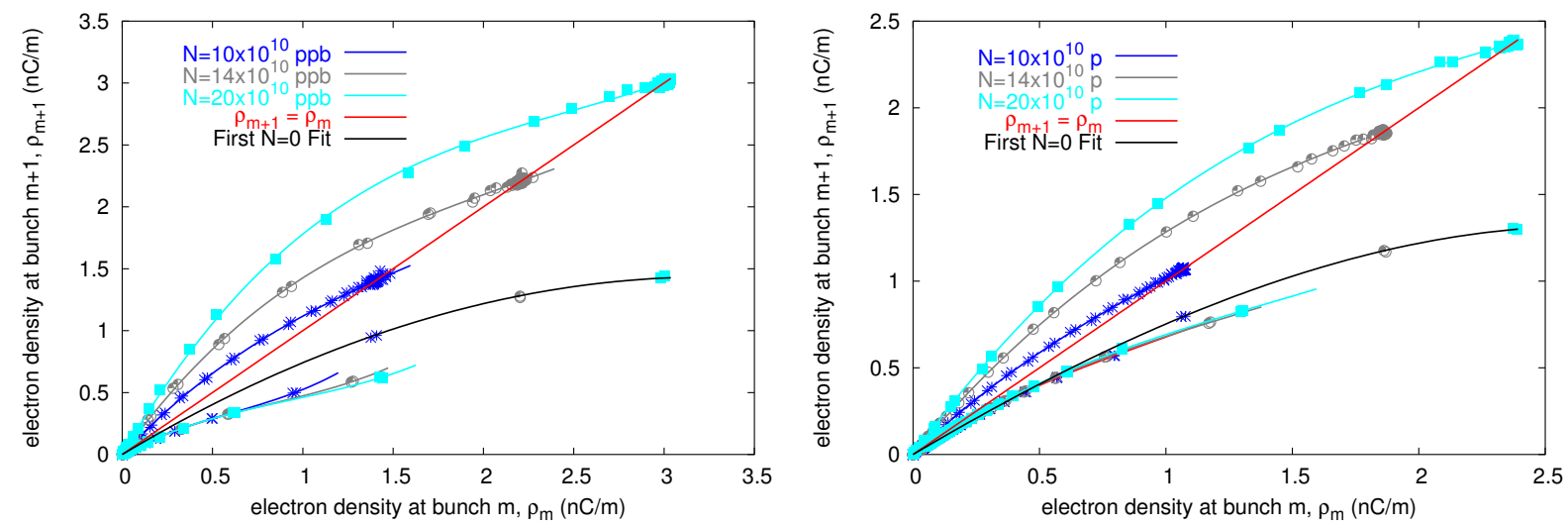

Figure 2: Average electron density after the bunch $m$ passes by, $\rho_{m+1}$, as a function of the electron density before the bunch $m$ passed by, $\rho_{m}$, for different bunch intensities, $N$. The left hand plot shows the $C S E C$ output, while the points on the right hand plot come from ECLOUD simulation. In both cases, the lines correspond to cubic fits applied to the average bunch to bunch points.

protons. For $N>8 \cdot 10^{10}$ protons, the linear coefficient, $a$, becomes larger than 1 for both ECLOUD and $C S E C$, and increases linearly in a first approximation. For large $N, a$ grows more slowly because the space charge effects have a faster influence: the more bunch intensity, the more electrons are created after a bunch passage, and therefore the space charge effects will be sooner noticeable. This is equivalent to say that the build up time tends to stabilize with increasing bunch intensities.

In all cases (different $N$ ), and using both codes, the quadratic coefficient, $b$, is negative to give concavity to the electron cloud density evolution in the space $\left(\rho_{m}, \rho_{m+1}\right)$. This coefficient decreases (increases in absolute value) for $C S E C$ results, showing that as increasing the bunch intensity, $N$, the space charge effects are gaining influence. However, using ECLOUD results, $b$ only decreases for bunch intensities $N>12 \cdot 10^{10}$ protons. It is surprising that $b$ is not a monotonic function of the bunch intensity.

The cubic coefficient, $c$, is associated with electrons created by other mechanisms than the electron multipacting. This term is positive, consistent with the expectations, and one order of magnitude smaller than the linear term for $N>10^{10}$ protons. However, both codes differ significantly for $N<10^{10}$ protons. The proximity to the bunch intensity threshold, and the different ways both codes compute other electron sources are associated with these difference.

The need for cubic terms is strong when large electron cloud densities are produced by large bunch intensities. Large electron cloud densities (not produced for the bunch intensities handled by RHIC) perhaps need higher order expressions. This fact suggests that the cubic map is the Taylor expansion of a more universal form, for example:

$$
\rho_{m+1}=\frac{a^{\prime} \cdot \rho_{m}}{1+b^{\prime} \cdot \rho_{m}}=a \rho_{m}+b \rho_{m}^{2}+c \rho_{m}^{3}+\ldots \quad .
$$

It is found that maps such as this do not improve on the cubic map, therefore we continue the analysis using cubic maps.

\section{Minimization of electron density at RHIC}

After experimental observations during Run-3 [15, 16, 17], it was found that the use of gaps along the bunch train can be useful against the build up of the electron cloud. Since the growth time is longer than the decay time (see, for example, Fig. 1) the goal is to find out a bunch pattern around the RHIC circumference that does not trigger the electron cloud, or minimizes the detrimental effects of the phenomenon. In the following we will use triplets of integer numbers $\left(k_{s}, k_{b}, k_{g}\right)$ to describe bunch patterns, where $k_{s}$ gives the bunch spacing in buckets, $k_{b}$ the number of bunches filled with that spacing, and $k_{g}$ the number of "phantom" bunches added, i.e. bunches that are not filled in and therefore create a gap. Changing patterns can then 

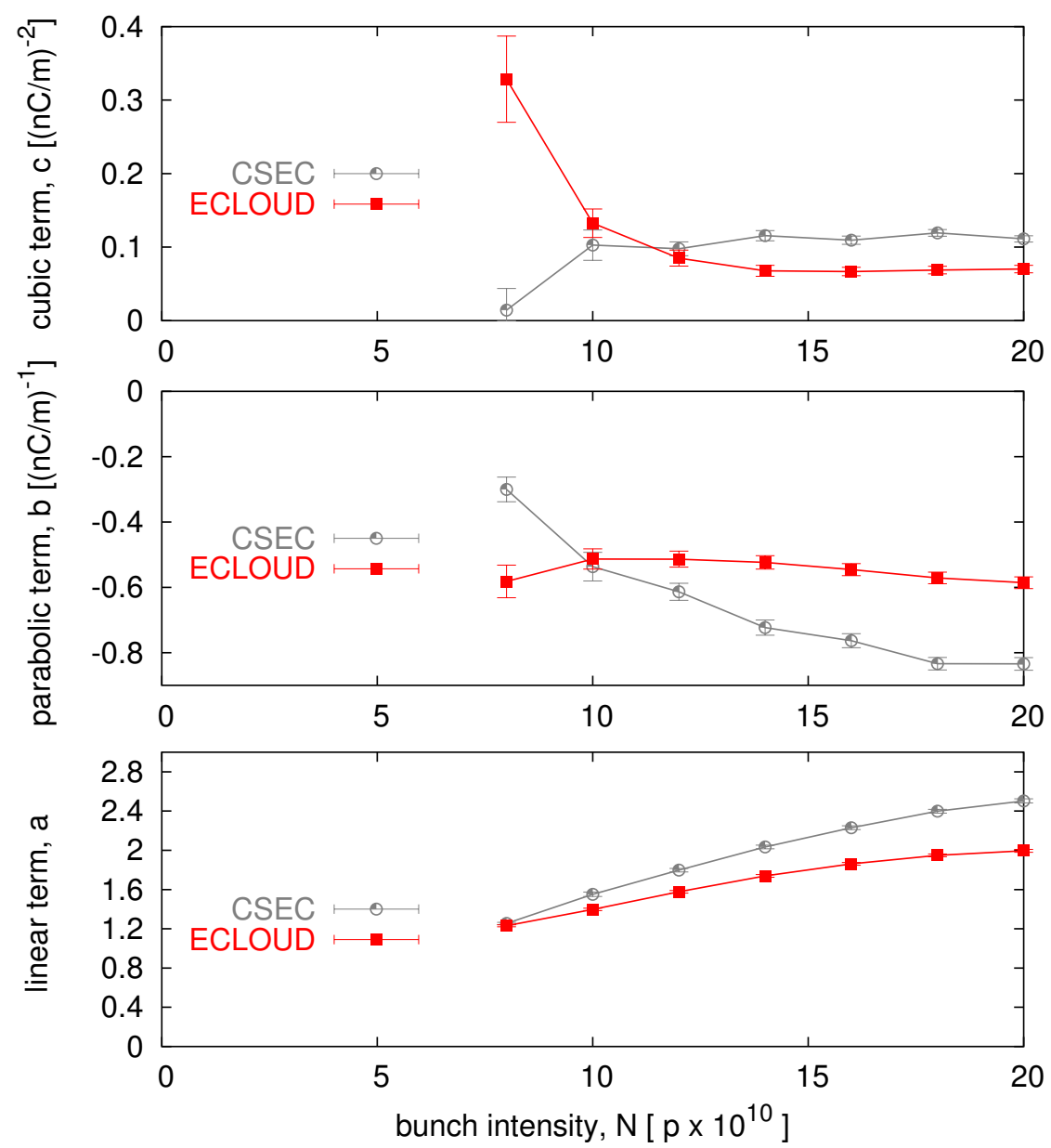

Figure 3: Evolution of the linear, $a$, parabolic, $b$, and cubic $c$ terms determining the electron cloud build up as a function of the bunch intensity, $N$, for both $E C L O U D$ (red squares) and $C S E C$ (grey points).

be described by adding a new triplet. For example the configuration $(2,2,1)(3,4,0)$ would correspond to the pattern

$1-0-1-0-0-0-1-0-0-1-0-0-1-0-0-1-0-0$

where 1 denotes a filled and 0 denotes an empty bucket. If not otherwise noted, it is assumed that a pattern repeats until the abort gap is reached. When using the $28 \mathrm{MHz}$ RF cavities, RHIC has an harmonic number of 360 buckets, and it is allowed to inject a bunch every 3 buckets (minimum) with an abort gap of 30 buckets. In terms of possible bunches, this implies a maximum of 110 bunches.

In the following, different distributions of 68 bunches are discussed. Figure 4 shows the result of three attempts in injecting three different bunch patterns: $(3,16,4),(3,12,8)$, and $(3,14,6)$. Even though pressure rises are detected for the first two cases and not for the third one, (see pressure rise at the blue section in Fig. 4, bottom), we can inject up to 68 bunches using the configuration $(3,12,8)$, whereas injection of bunch pattern $(3,14,6)$ cannot be completed. A comparison among the three cases is complicated by the fact that bunch intensity and bunch length are not the same for all fills. Figure 4 also shows that the attempt to fill bunch pattern $(3,14,6)$ was successful, but it is not taken into account for this study because the bunch length was the double of the previous attempted fills. Bunch intensity and length are comparable for fills $(3,16,4)$ and $(3,12,8)$, which also show similar vacuum behavior. On the other hand, fill $(3,14,6)$ has bunch lengths that are larger by a factor of 2 compared to previous attempts. This, together with the reduced bunch intensity, can account for the suppression of ECs. Table 2 summarizes the characteristics of the different cases and compares the relative luminosity.

Reference [15] studies the effect of the bunch pattern on the Electron Cloud and pressure rise. Several 
Table 2: Comparison of bunch patterns tested in RHIC at injection.

\begin{tabular}{lccccc}
\hline \hline parameter & unit & $\begin{array}{c}\text { reference } \\
\text { case }\end{array}$ & $\begin{array}{c}\text { fill } \\
\text { no } 1\end{array}$ & $\begin{array}{c}\text { fill } \\
\text { no 2 }\end{array}$ & $\begin{array}{c}\text { fill } \\
\text { no 3 }\end{array}$ \\
\hline bunch pattern & $\ldots$ & $(6,1,0)$ & $(3,16,4)$ & $(3,12,8)$ & $(3,14,6)$ \\
no of bunches & $\ldots$ & 56 & 41 & 69 & 78 \\
average bunch intensity $N_{b}$ & $10^{11} \mathrm{p}$ & 1.0 & 1.1 & 1.0 & 0.9 \\
total intensity & $10^{11} \mathrm{p}$ & 56.0 & 44.3 & 68.1 & 70.2 \\
full bunch length & $\mathrm{ns}$ & $\ldots$ & 16.5 & 17.6 & 34.2 \\
pressure rise & $\ldots$ & $\ldots$ & yes & yes & no \\
luminosity scaling factor & $\ldots$ & 1.00 & 0.88 & 1.23 & 1.13 \\
\hline \hline
\end{tabular}

computer simulation runs were launched with different bunch patterns. The criteria to minimize the effects of the electron cloud were the comparison between the average and the maximum value of the electron density created by each bunch pattern. The conclusion, in agreement with the way B-factories work, is that the most sparse distribution of bunches is the best way to optimize luminosity. However, assuming that one $C S E C$ run takes $\approx 1 \mathrm{~h}$, if we want to study all the possibilities of distributing 68 bunches in 110 possible buckets, it is obvious that we cannot simulate

$$
\frac{110 !}{(110-68) ! \cdot 68 !} \approx 10^{30}
$$

different bunch distributions. In this section, we will show how maps elegantly answer the initial question: given a fixed beam intensity, what is the optimum bunch distribution along the bunch train to minimize the electron cloud density?
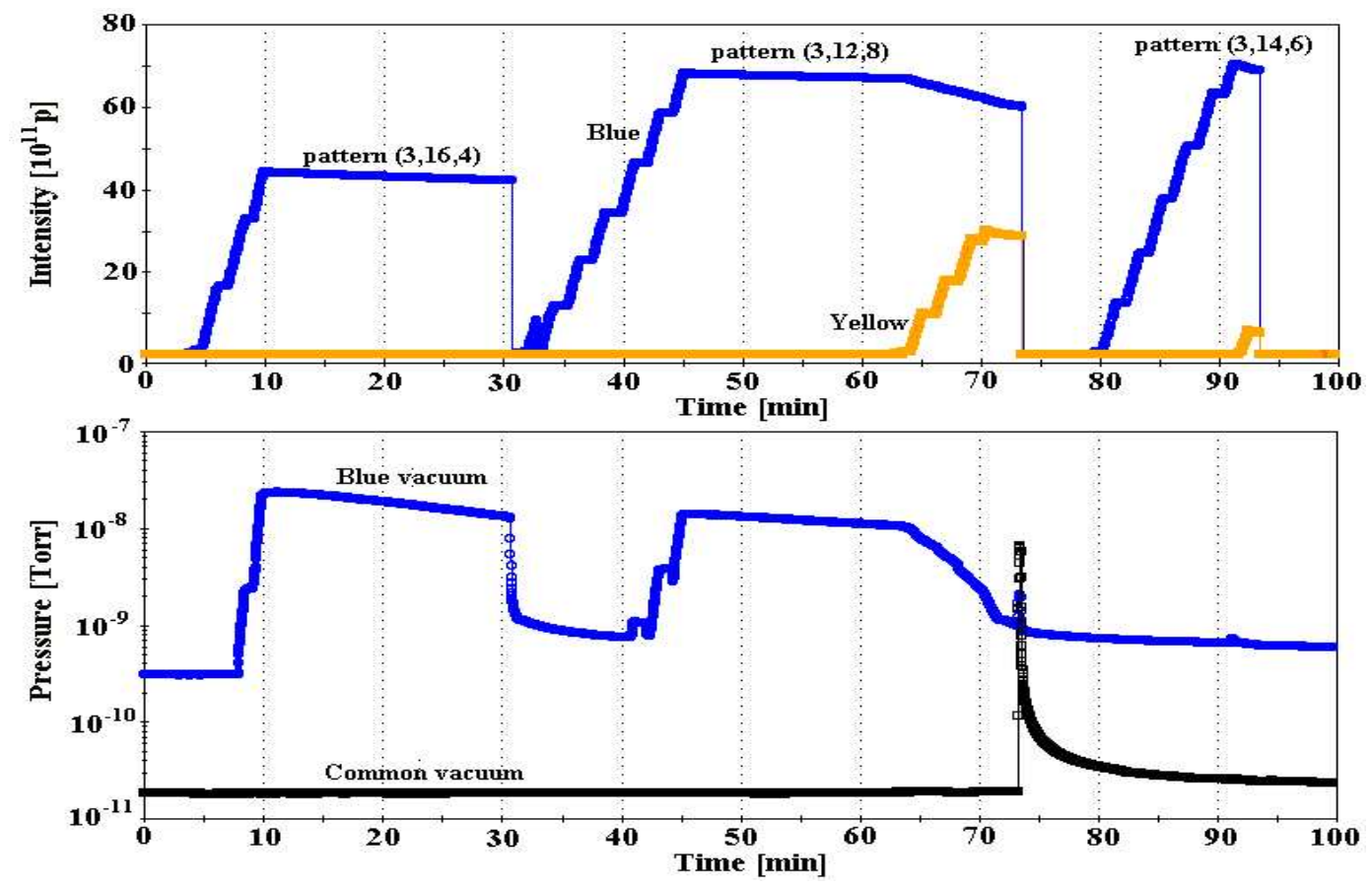

Figure 4: Attempt of filling RHIC with three different bunch patterns: $(3,16,4),(3,12,8)$, and $(3,14,6)$. The top plot shows the total intensity in the ring, while the bottom plot shows the pressure in one of the unbaked warm regions at RHIC (blue vacuum). Unlike the first attempt, $(3,16,4)$, the injection in the second case, $(3,12,8)$, does not prevent machine operation although the pressure rise is noticeable. The third case, $(3,14,6)$ is not taken into account due to an unusually large bunch spacing. 


\section{1 $\quad$ First $N_{m}=0$ and $N_{m}=1$}

The bunch to bunch simulations carried out with CSEC and reported in [15] are reproduced and compared using $M E C$ (Maps for Electron Cloud). The parameters used in all cases are shown in Table 1. The use of $M E C$ is divided in four cases, depending on the bunch charge of the bunch $m$ passing by:

- "Full" bunches, which in this case denote bunches with charge $N=8 \cdot 10^{10}$ protons. The cubic form is similar to Eq. 6, and the coefficients are denoted using the vector $\vec{A}_{11}=\left(a_{11}, b_{11}, c_{11}\right)$.

- "Empty" bunches, which denote bunches with bunch charge $N=0$. In this case the corresponding cubic form is obtained from the decay case, and it is denoted with the vector $\vec{A}_{00}=\left(a_{00}, b_{00}, c_{00}\right)$.

- First "empty" bunch, which denotes an empty bunch after a populated bunch, i.e. $N_{m}=0$ and $N_{m-1}=1$. The corresponding cubic form is denoted with the vector: $\vec{A}_{01}=\left(a_{01}, b_{01}, c_{01}\right)$.

- First "full" bunch, which denotes a full bunch after an empty one, i.e. $N_{m}=1$ and $N_{m-1}=0$. The corresponding cubic form is denoted with the vector: $\vec{A}_{10}=\left(a_{10}, b_{10}, c_{10}\right)$.

The need of this subdivision requires analysis of two figures: in Fig. 2 one can see that the "first $N_{m}=0$ " is out of the evolution of decay curve, i.e. the curve corresponding to "phantom" bunches.

Figure 5 justifies the case for the "first $N_{m}=1$ " curve. Figure 5 evidences that the transition from "empty" to "full" also requires two bunches, in the same way that the transition from "full" bunch to "empty" bunch is done in two bunches. One obtains successful results when comparing the bunch to bunch evolution using $C S E C$ and $M E C$ : see the graphical comparison between the bunch to bunch evolution using the two codes at Figures 6, 7, 8, and 9. Table 3 compares numerically the maximum and average values for the linear electron cloud density at the last turn following the $n s$ to $n s$ evolution in [15], and the bunch to bunch evolution using $C S E C$ and $M E C$. The largest difference is between a $15 \%$ for the maximum density (corresponding to the case $(3,2,0)(6,4,0)$ ), while for the average density the maximum difference is within an error bar of $17 \%$, corresponding to the case $(3,23,17)$. While $C S E C$ uses about $\approx 1 h \mathrm{CPU}$ time for each case, $M E C$ is obviously much faster and only uses $\approx 1 \mathrm{~ms}$, which represents a speed up of seven orders of magnitude.

Table 3: Maximum, $\rho_{\max }$, and average, $\rho_{\text {avg }}$, bunch to bunch values of the linear electron density computed with $C S E C$ and $M E C$ for two different bunch patterns. The results agree within a range of about a $15 \%$. This error bar includes the results from analysis at Ref. [15].

\begin{tabular}{lcccccc}
\hline \hline parameter & unit & $\begin{array}{c}\text { case } \\
\text { no } 1\end{array}$ & $\begin{array}{c}\text { case } \\
\text { no } 2\end{array}$ & $\begin{array}{c}\text { case } \\
\text { no } 3\end{array}$ & $\begin{array}{c}\text { case } \\
\text { no } 4\end{array}$ & $\begin{array}{c}\text { case } \\
\text { no } 5\end{array}$ \\
\hline bunch pattern & $\ldots$ & $(3,68,52)$ & $(3,23,17)$ & $(3,12,8)$ & $(3,4,0)(6,8,0)$ & $(3,2,0)(6,4,0)$ \\
\# of bunches & $\ldots$ & 68 & 68 & 68 & 68 & 68 \\
bunch intensity, $N$ & $10^{10} \mathrm{p}$ & 8.0 & 8.0 & 8.0 & 8.0 & 8.0 \\
$\rho_{\max }$ using $C S E C$ & $\mathrm{nC} / \mathrm{m}$ & 0.8991 & 0.6203 & 0.2849 & 0.2221 & 0.2033 \\
$\rho_{\max }$ using $M E C$ & $\mathrm{nC} / \mathrm{m}$ & 0.9302 & 0.6645 & 0.2861 & 0.2184 & 0.2370 \\
$\rho_{\text {ave }}$ using $C S E C$ & $\mathrm{nC} / \mathrm{m}$ & 0.3023 & 0.1433 & 0.0981 & 0.1006 & 0.0922 \\
$\rho_{\text {ave }}$ using $M E C$ & $\mathrm{nC} / \mathrm{m}$ & 0.3216 & 0.1156 & 0.1045 & 0.0992 & 0.0924 \\
\hline \hline
\end{tabular}




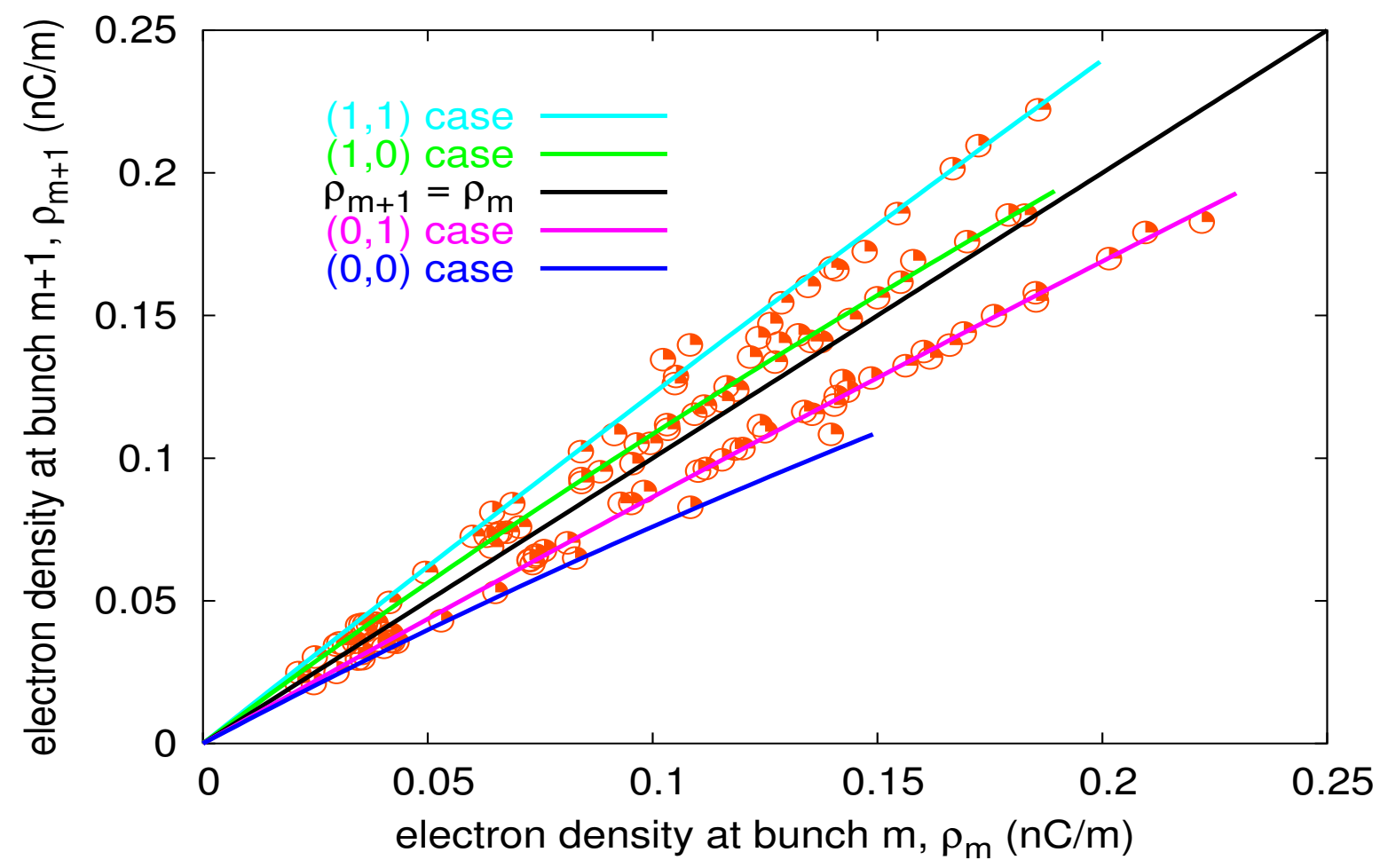

Figure 5: Electron cloud density in the $\left(\rho_{m}, \rho_{m+1}\right)$ for the bunch pattern $(3,4,0)(6,8,0)$. The plot shows that four different behaviors are required: the case $(1,1)$ refers to "full" bunches preceded by another "full" bunch; the case $(1,0)$ refers to "full" bunches preceded by a "phantom" bunch; the case $(0,1)$ to "empty" bunches preceded by a "full" bunch; while the case $(0,0)$ denotes a "phantom" bunch preceded by another "phantom" bunch. 


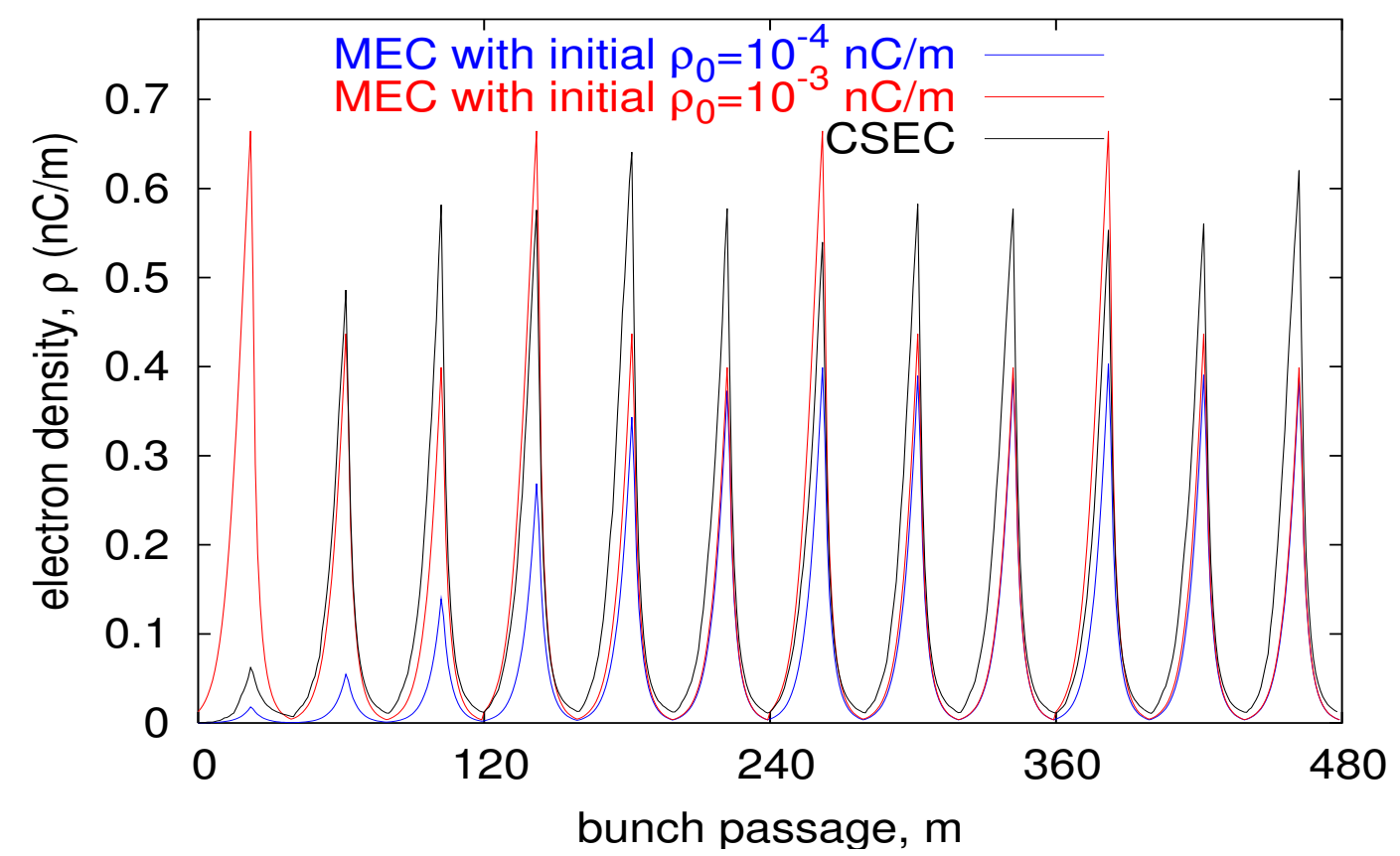

Figure 6: Electron cloud density evolution for bunch pattern (3,23.17) using $C S E C$ (black trace) and $M E C$ with two different initial electron densities: $\rho_{0}=10^{-4} \mathrm{nC} / \mathrm{m}$ (blue line) and $\rho_{0}=10^{-3} \mathrm{nC} / \mathrm{m}$ (red line). No matter the initial electron density, $M E C$ results agree for the last turn (from bunch passage 360 to 480) within an acceptable error range.

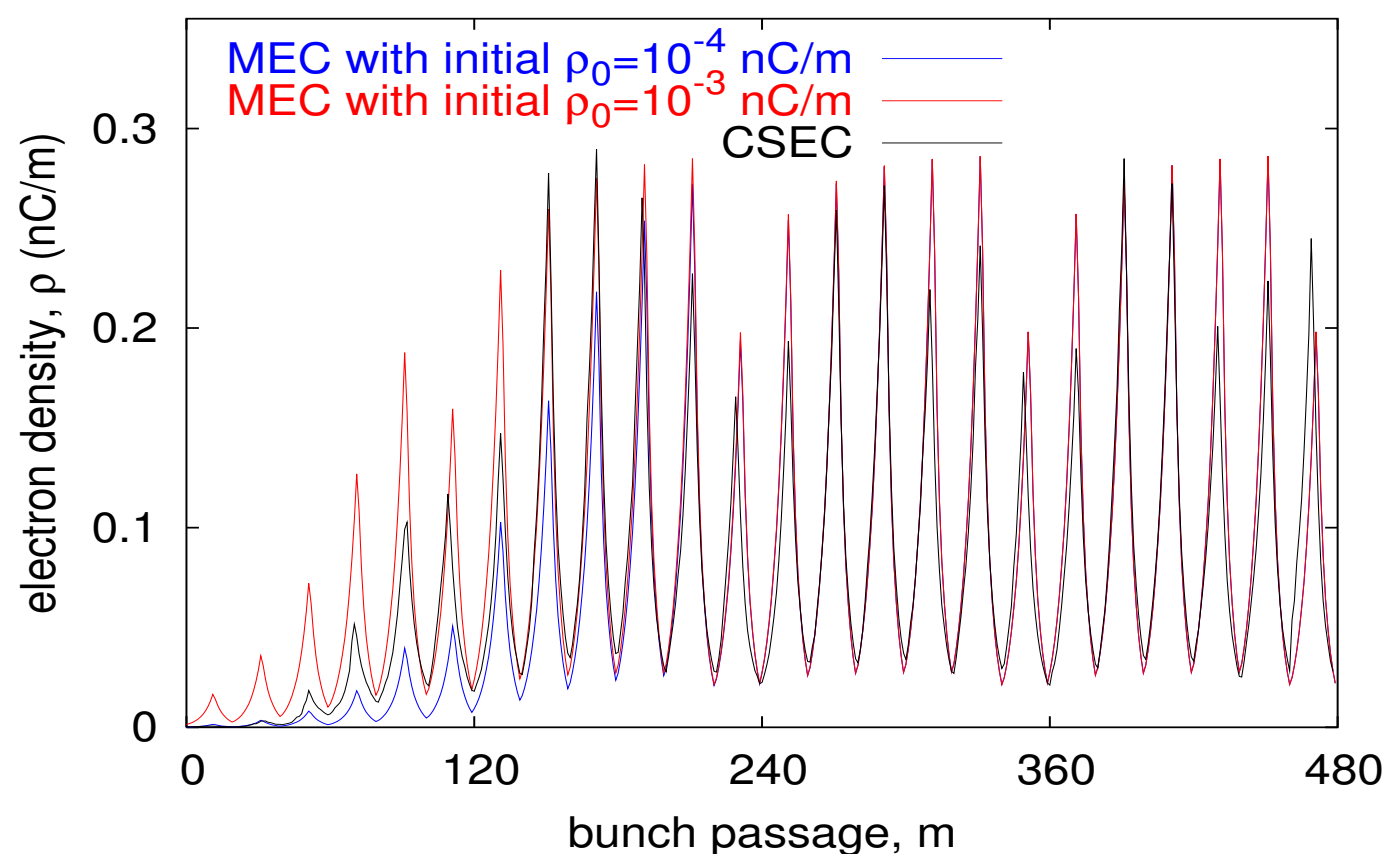

Figure 7: Electron cloud density evolution for bunch pattern $(3,12,8)$ using $C S E C$ (black trace) and $M E C$ with two different initial electron densities: $\rho_{0}=10^{-4} \mathrm{nC} / \mathrm{m}$ (blue line) and $\rho_{0}=10^{-3} \mathrm{nC} / \mathrm{m}$ (red line). No matter the initial electron density, $M E C$ results agree for the last turn (from bunch passage 360 to 480) within an acceptable error range. 


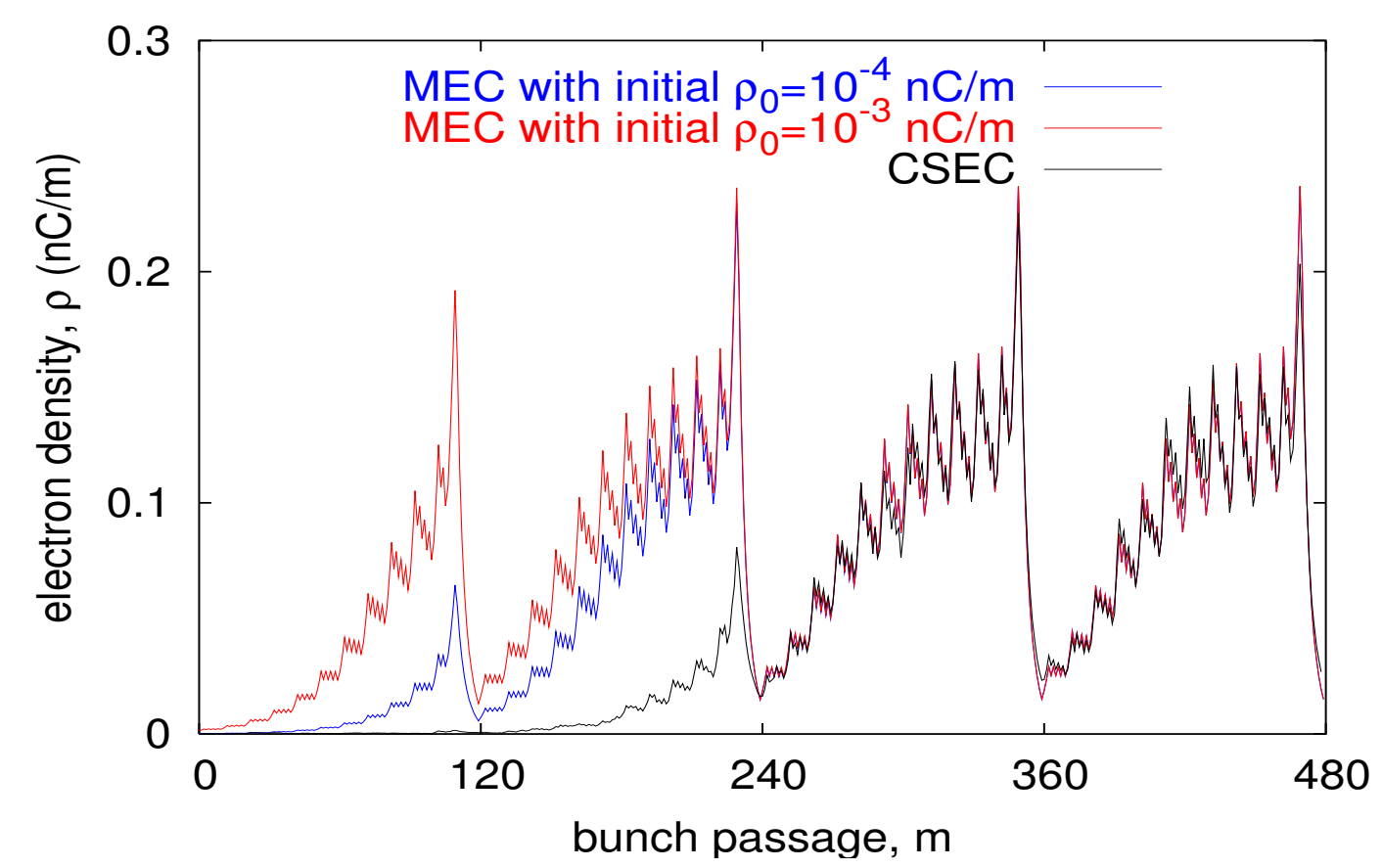

Figure 8: Electron cloud density evolution for bunch pattern $(3,2,0)(6,4,0)$ using $C S E C$ (black trace) and $M E C$ with two different initial electron densities: $\rho_{0}=10^{-4} \mathrm{nC} / \mathrm{m}$ (blue line) and $\rho_{0}=10^{-3} \mathrm{nC} / \mathrm{m}$ (red line). No matter the initial electron density, $M E C$ results agree for the last turn (from bunch passage 360 to 480) within an acceptable error range.

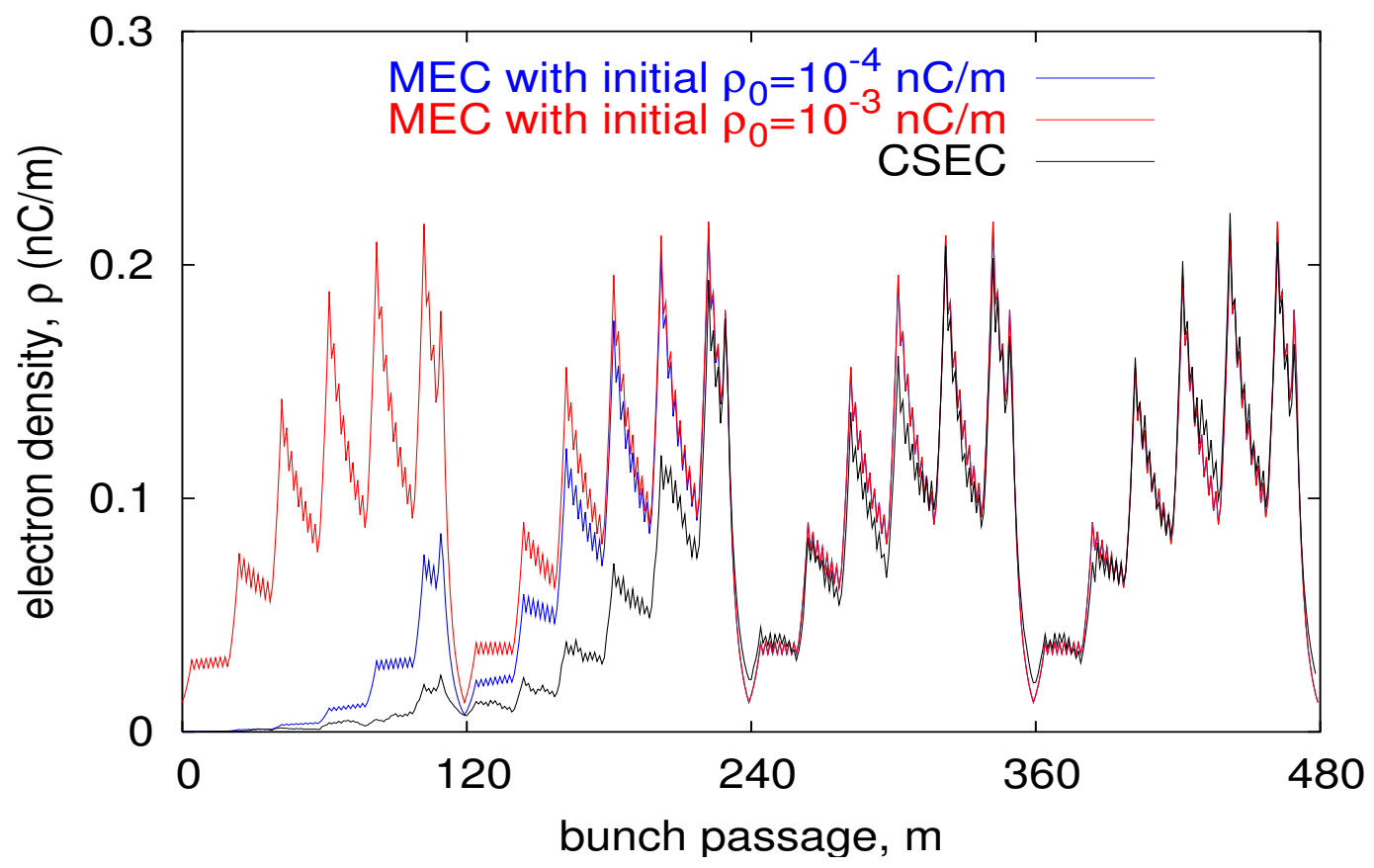

Figure 9: Electron cloud density evolution for bunch pattern $(3,4,0)(6,8,0)$ using $C S E C$ (black trace) and $M E C$ with two different initial electron densities: $\rho_{0}=10^{-4} \mathrm{nC} / \mathrm{m}$ (blue line) and $\rho_{0}=10^{-3} \mathrm{nC} / \mathrm{m}$ (red line). No matter the initial electron density, $M E C$ results agree for the last turn (from bunch passage 360 to 480) within an acceptable error range. 


\subsection{The linear approximation}

Four sets of polynomial coefficients, $\vec{A}_{11}(N), \vec{A}_{01}(N), \vec{A}_{00}(N)$, and $\vec{A}_{10}(N)$, are required to follow the bunch to bunch evolution of the electron cloud density. Figure 5 suggests that for small electron densities, its bunch to bunch evolution can be considered as linear in the $\left(\rho_{m}, \rho_{m+1}\right)$ space. If there is a total number of $M$ bunches in a ring with a "bunch harmonic" number of $H$, the linearization of the problem gives a one turn map that is simply:

$$
\rho_{m+H} \approx F(N) \rho_{m}
$$

where the "one turn factor"

$$
F \equiv\left(a_{10} a_{01}\right)^{i} a_{11}^{M-i} a_{00}^{H-M-i}
$$

and $i$ is the number of transitions from full to empty (and empty to full) bunches. In general the minimum possible number of transitions is $i=1$ (if all the bunches are clumped together), and the maximum number of transitions is the smaller of $M$ and $H-M$ (when the bunches are spread as sparsely as possible). The special case $i=0$ applies when there is no abort gap, $M=H$.

It is clear that if $F>1$ then the electron cloud density increases (to some saturated value), while if $F<1$ then the cloud disappears. When the one turn factor is rewritten as

$$
F=\left(\frac{a_{10} a_{01}}{a_{11} a_{00}}\right)^{i}\left(\frac{a_{11}}{a_{00}}\right)^{M} a_{00}^{H}
$$

it is clear that, for given $M$ and $H$ and $N$, the smallest (largest) value of $F$ occurs for the largest (smallest) allowed value of $i$ if

$$
\left(\frac{a_{10} a_{01}}{a_{11} a_{00}}\right)<1
$$

and vice versa. Since Eq. 11 is valid for RHIC parameters, which shows that either the most sparse distribution of a fixed number of fixed population bunches is the most stable against electron cloud growth.

Thus, from the mapping approach and using standard maths we analytically demonstrate that the most sparse distribution of bunches in a ring circumference minimizes the detrimental effects of the multi-bunch electron cloud effects. This is not a big surprise if we consider the possibility of evenly distributed bunches, i.e. the same bunch spacing between all bunches. In this case, the most sparse distribution of bunches is equivalent to use the larger bunch spacing between them. However, Eq. 10 demonstrates that this idea is also valid for unevenly spaced bunches along a bunch train.

\section{Conclusion}

The multi-bunch electron cloud build-up at RHIC can be determined using a third order polynomial map, written as $\vec{A}=(a, b, c)$. For a given beam pipe, these coefficients are a function of the beam parameters. The dependence of these parameters $(a, b, c)$ on the bunch intensity, $N$ for can be derived from electron cloud simulations codes, like CSEC or ECLOUD.

A memory of "two bunches" is found to be necessary when jumping back and forth from full to empty bunches, and therefore a complete algorithm requires four vectors: $\vec{A}_{11}, \vec{A}_{10}, \vec{A} 00$, and $\vec{A}_{01}$. A simulation program, $M E C$ uses these vectors, for example to find out how to minimize the effects of the electron cloud given a machine limitation using alternative bunch patterns. Actual values for the vectors analytically demonstrate that the most sparse distribution of bunches (even when they cannot be evenly spaced) is the most stable against electron cloud effects.

In the future, and in order to obtain a better understanding of the problem, it is desirable to explore how the polynomial coefficients vary as a function of the physical parameters influencing the electron cloud (SEY, chamber dimensions, bunch spacing, bunch charge, et cetera). Application of maps to other machines (specially the B-Factories) is also interesting to study the universality of map formalism.

\section{Acknowledgments}

We are very grateful for discussions with, and support from, Mike Blaskiewicz, Angelika Drees, Wolfram Fischer, H.C. Hseuh, Nick Luciano, Giovanni Rumolo (GSI), Rogelio Tomás, Dejan Trbojevic, Lanfa Wang, and S.Y. Zhang. 


\section{References}

[1] P. Farnsworth. J. Franklin Inst, Vol. 218, Issue 4, 411-444, October 1934

[2] G. I. Budker, G. I. Dimov, and V. G. Dudnikov, in Proceedings of the International Symposium on Electron and Positron Storage Rings,Saclay, 1966 (Universitaires De France, Orsay, 1966), p. VIII-6-1.

[3] O. Gröbner, in Proceedings of the 10th International Conference on High Energy Accelerators, Serpukhov, 1977 (USSR Academy of Science, Moscow, 1977), p. 277.

[4] Review of Single-Bunch Instabilities Driven by an Electron Cloud F. Zimmermann, Presentation at ECLOUD'04. Napa, USA, April 2004.

[5] http://wwwslap.cern.ch/collective/ecloud02/ecsim/index.html

[6] http://wwwslap.cern.ch/collective/ecloud02

[7] L. Wang, private communications.

[8] G. Rumolo, private communications.

[9] M. Blaskiewicz, private communications.

[10] Can low energy electrons affect high energy accelerators? R. Cimino, I. Collins, et al. CERN-AB-2004012-ABP, Feb.2004.

[11] Microscopic probabilistic model for the simulation of secondary electron emission, M.A. Furman and M. Pivi, PRST-AB 5, 124404, Dec.2002.

[12] Electron cloud measurements and simulations for the Brookhaven Relativistic Heavy Ion Collider, W. Fischer, M. Blaskiewicz, M. Brennan, T. Satogata, PRST-AB 5, 124401 (2002).

[13] Photoelectrons and Multipacting in the LHC : Electron Cloud Build-up G. Stupakov, CERN-LHCProject-Report-141, Geneva, Oct 1997.

[14] Electron cloud phase transitions, U. Iriso and S. Peggs. C-AD/AP/147, April 2004.

[15] Bunch patterns and pressure rise in RHIC, W. Fischer and U. Iriso, C-AD/AP/118, October 2003.

[16] Electron Cloud and pressure rise simulations for RHIC, U. Iriso et al, MPPE025, Proceedings of PAC'03, Portland, May 2003.

[17] RHIC Pressure Rise and Electron Cloud, S.Y. Zhang et al. MOPA010, Proceedings of PAC'03, Portland, May 2003. 